\title{
Low-voltage closed loop MEMS actuators
}

\section{Shahrzad Towfighian, Glenn R. Heppler \& Eihab M. Abdel-Rahaman}

\section{Nonlinear Dynamics}

An International Journal of Nonlinear Dynamics and Chaos in Engineering Systems

ISSN 0924-090X

Volume 69

Combined 1-2

Nonlinear Dyn (2012) 69:565-575

DOI 10.1007/s11071-011-0287-9
ISSN 0924-090X

\section{Nonlinear Dynamios}

An International Journal of Nonlinear Dynamics and Chaos in Engineering Systems

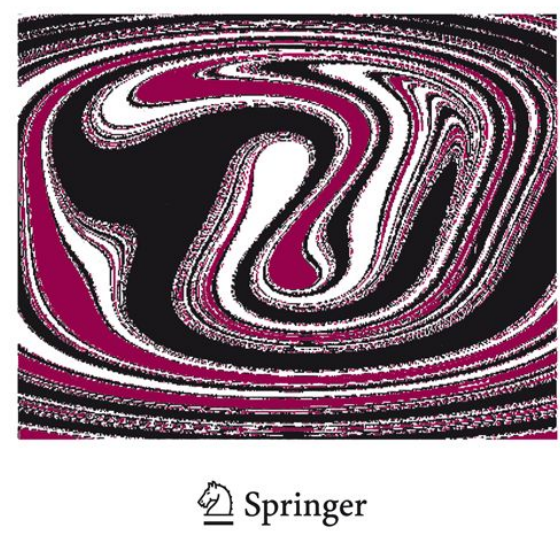


Your article is protected by copyright and all rights are held exclusively by Springer Science+Business Media B.V.. This e-offprint is for personal use only and shall not be selfarchived in electronic repositories. If you wish to self-archive your work, please use the accepted author's version for posting to your own website or your institution's repository. You may further deposit the accepted author's version on a funder's repository at a funder's request, provided it is not made publicly available until 12 months after publication. 


\title{
Low-voltage closed loop MEMS actuators
}

\author{
Shahrzad Towfighian • Glenn R. Heppler • \\ Eihab M. Abdel-Rahaman
}

Received: 23 March 2011 / Accepted: 26 November 2011 / Published online: 22 December 2011

(C) Springer Science+Business Media B.V. 2011

\begin{abstract}
An efficient electrostatic resonator is designed by adding a low voltage controller to an electrostatic actuator. The closed loop actuator shows stable, and bi-sable behaviors with bounded chaotic oscillations as large as $117 \%$ of the capacitor gap. The controller voltage is decreased from a previously designed resonator to less than $9 \mathrm{~V}$ thereby reducing the load on the controller circuit components. Bifurcation diagrams are obtained showing the frequency and magnitude of AC voltage required for chaotic oscillations to develop. The information entropy, a measure of chaotic characteristic, is calculated for the microresonator and is found to be 0.732 .
\end{abstract}

Keywords Micro-resonator - Electrostatic actuator . Closed loop actuator

S. Towfighian $(\bowtie)$

University of Toronto, 5 King's College Rd., Toronto, ON, M5S 3G8, Canada

e-mail: sherryt@mie.utoronto.ca

G.R. Heppler · E.M. Abdel-Rahaman

University of Waterloo, 200 University Avenue West,

Waterloo, Ontario, Canada N2L 3G1

G.R. Heppler

e-mail: heppler@uwaterloo.ca

E.M. Abdel-Rahaman

e-mail: eihab@uwaterloo.ca

\section{Introduction}

Appearance of chaos is not usually desired and there are numerous control systems designed to avoid it. On the other hand, there are reports on the constructive use of chaos in macro-systems for structural health monitoring of aero-elastic systems [1-3], fault detection in roller bearings [4], and detection of corrosion [5]. Circuit chaotic oscillations have also been used for secure communications [6].

Despite the few reported studies on the usefulness of chaos in macro-scale applications, there is no evidence of applying chaos for detection in micro-scale systems. The main reason for this deficiency is the lack of resources on the non-linear dynamic behavior of MEMS. Most researchers avoid non-linear systems due to their complexity. Only in the last decade, have researchers paid attention to the study of nonlinear dynamics in MEMS and NEMS since the main elements of these systems, including cantilever beams and doubly clamped beams, show significant nonlinear responses that cannot be neglected and need thorough study [7]. Such attention is now growing due to the fast growing area of NEMS showing strong chaotic responses that can be effectively used toward developing non-linear sensors with improved resolution.

In smaller scale NEMS, there are recent studies on non-linear vibration, chaos, and their applications [8-10]. Bucks et al. [8] found higher mass detection sensitivity is achieved once a nano-mechanical res- 
onator is operated in the non-linear region. Conley et al. [9] studied the onset of non-linear planar motion and non-planar whirling motion of electrostatically excited nano-wires with a proposed application in an overload detection mechanism. This study was further developed by Chen et al. [10], who presented a bifurcation diagram for the extensive non-planar chaotic oscillations of a nano-wire at elevated AC voltages. Chen et al. [10] suggested using the chaotic nano-wire oscillators in random number generation used in secure communications.

A bi-stable system with two stable equilibrium points is a typical system used to produce chaotic oscillations. Bi-stability and chaos have been reported in atomic force microscopy (AFM) [11-16], and electrostatic MEMS [17-29]. Bi-stability in MEMS was analyzed for electrostatic actuators with geometrical nonlinearities [21-24]. Zhang et al. [21] investigated the static response of an arch-shaped beam that showed either snap-through and pull-in, or only pull-in, under electrostatic loading. The snap-through response led to bi-stability with a large amplitude response. Nonlinear dynamic responses of arches including softening behavior, dynamic pull-in, and snap through were recently reported by Younis et al. [28] and Ouakad et al. [29]. Bi-stability has also been reported in static response and hysteretic jumps in the response of AFM when the tip-sample distance was less than $10 \mathrm{~nm}$ [14]. The potential energy in the bi-stable region was an asymmetric two-well potential. They also found that the frequency response at large amplitudes contained multiple harmonics due to period doubling. Zhang et al. [26] studied softening in an electrostatic microcantilever beam and reported period doubling bifurcations in their simulations.

Chaotic vibrations have been observed in bi-stable non-interdigitated comb drive electrostatic actuators. Wang et al. [18] modeled them using a mass-spring system as a Duffing oscillator with a two-well potential field. Through simulations and experiments they have shown chaotic oscillations in the two-well region of the system. As an extension to this work, De and Aluru [19] presented a model for the system including the electrostatic forcing, non-linear stiffness, and squeeze film damping. They showed that even in the absence of the non-linear mechanical and fluidic forces, the system had chaotic attractors. Thus, the electrostatic forces were found to be the primary mechanism producing chaos. The boundaries for chaotic motion in the amplitude-frequency space of the applied voltage were found both from experiments and from Melnikov's method by DeMartini et al. [20].

Chaos has also been studied in a closed loop feedback controlled electrostatic actuator with capacitive sensing [27] designed by $\mathrm{Lu}$ and Fedder [30] for position tracking of probe-based magnetic disk drives. Other approaches for controlling electrostatic actuators include using delayed feedback controllers [31,32]. Liu et al. [27] used a lumped mass model to study the probe response and reported chaotic oscillation in the presence of forced excitations. A comprehensive study of the nonlinear system dynamics was carried out by Towfighian et al. [33] to investigate parameters space of the actuator for stable and bi-stable behaviors, and chaotic oscillations.

In this study, a quadratic control law [34] is introduced for the electrostatic micro-beam actuator to create an efficient resonator. The proposed resonator requires less voltage and produces larger motions compared to that previously reported [33].

\section{Actuator model}

The configuration of the electrostatic actuator is shown in Fig. 1 and is comprised of a cantilever beam above a fixed electrode and a voltage regulator. The equation of motion for the micro-beam (Fig. 2) using an EulerBernoulli model is

$$
\begin{aligned}
\rho & A \frac{\partial^{2} \hat{w}(\hat{x}, \hat{t})}{\partial \hat{t}^{2}}+E I \frac{\partial^{4} \hat{w}(\hat{x}, \hat{t})}{\partial \hat{x}^{4}}+c \frac{\partial \hat{w}(\hat{x}, \hat{t})}{\partial \hat{t}} \\
& =\frac{\varepsilon_{0} b V^{2}}{2(d-\hat{w}(\hat{x}, \hat{t}))^{2}}
\end{aligned}
$$

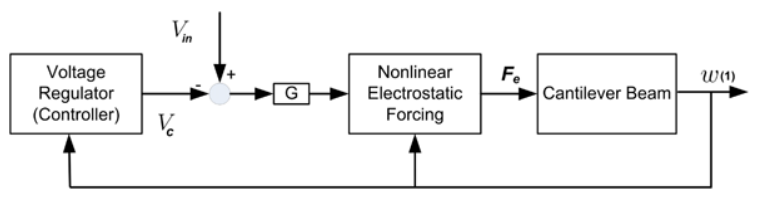

Fig. 1 Closed loop system

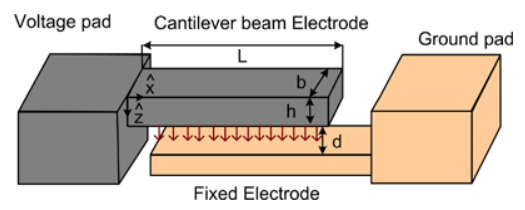

Fig. 2 Schematic of the micro-beam oscillator 
Table 1 Actuator parameters

\begin{tabular}{lll}
\hline Parameter & Symbol & Value \\
\hline Density & $\rho$ & $2331 \frac{\mathrm{kg}}{\mathrm{m}^{3}}$ \\
Beam Length & $L$ & $157.4 \mu \mathrm{m}$ \\
Beam Width & $b$ & $10 \mu \mathrm{m}$ \\
Beam Thickness & $h$ & $1.9 \mu \mathrm{m}$ \\
Initial Gap & $d$ & $1.9 \mu \mathrm{m}$ \\
Non-dimensional Damping Coefficient & $\mu$ & 0.6153 \\
Permittivity of Free Space & $\varepsilon_{0}$ & $8.85 \mathrm{E}-12 \frac{\mathrm{F}}{\mathrm{m}}$ \\
Modulus of Elasticity & $E$ & $150 \mathrm{GPa}$ \\
Integrator Gain & $K_{I} / T$ & $0.1776 \frac{1}{\mathrm{~s}}$ \\
\hline
\end{tabular}

where $\hat{w}(\hat{x}, \hat{t})$ is the deflection of the beam in the transverse $\hat{z}$ direction, $\hat{x}$ is the coordinate along the beam length, and $\hat{t}$ is time. The linear viscous damping coefficient per unit length $c$ is used to account for damping losses due to the beam motion through air, $V$ is the applied voltage, $A$ is the cross sectional area, $I$ is the area moment of inertia, and the other parameters are defined in Table 1 . To simplify the notation, $\hat{w}(x, t)$ is shown as $\hat{w}$. Multiplying both sides of the equation by the denominator of the electrostatic force and using the non-dimensional parameters

$x=\frac{\hat{x}}{L}, \quad w=\frac{\hat{w}}{d}, \quad t=\frac{\hat{t}}{T}$

where $L$ is the beam length, and

$T=\sqrt{\frac{\rho A L^{4}}{E I}}$.

Equation (1) is rewritten as

$$
\begin{aligned}
& \frac{\partial^{2} w}{\partial t^{2}}(1-w)^{2}+\frac{\partial^{4} w}{\partial x^{4}}(1-w)^{2}+\mu \frac{\partial w}{\partial t}(1-w)^{2} \\
& \quad=\alpha V^{2}
\end{aligned}
$$

where

$\mu=\frac{c L^{4}}{E I T}, \quad \alpha=\frac{\varepsilon_{0} b L^{4}}{2 E I d^{3}}$.

The normalized partial differential equation of motion of the closed-loop actuator is

$$
\begin{aligned}
& \ddot{w}(1-w)^{2}+w^{(4)}(1-w)^{2}+\mu \dot{w}(1-w)^{2} \\
& \quad=\alpha\left(V_{\text {in }}-V_{c}\right)^{2}
\end{aligned}
$$

where $V_{\text {in }}$ is the system voltage input, and $V_{c}$ is the controller voltage. To obtain the static and dynamic responses of the actuator, the partial differential equation of motion in (6) is converted to an ordinary differential equation using separation of variables and a one-mode Galerkin's expansion as described in [33]. Two ordinary differential equations are thus obtained:

$$
\left\{\begin{array}{c}
\left(\ddot{q}+\mu \dot{q}+\omega_{1}^{2} q\right)\left(1+c_{1} q+c_{2} q^{2}\right) \\
=c_{3} \alpha\left(V_{\text {in }}-V_{c}\right)^{2}, \\
\dot{V}_{c}=-K_{I}\left(V_{c}-\frac{a_{0}+a_{1} q+a_{2} q^{2}}{\sqrt{\alpha}}\right)
\end{array}\right.
$$

where $q$ is the normalized beam tip deflection, $\omega_{1}$ is the first natural frequency of the beam, and $c_{1}, c_{2}$, and $c_{3}$ are found from applying Galerkin's method. The second equation represents the quadratic controller law with a constant integrator gain $K_{I}$. The parameters $a_{0} ; a_{1} ; a_{2}$ in the controller equation are constants chosen to obtain four equilibrium points for a voltage range as explained in Sect. 3 .

To implement the electronic circuit of the controller, the second equation is rewritten to include dimensional time:

$\dot{V}_{c}=-\frac{K_{I}}{T}\left(V_{c}-\frac{a_{0}+a_{1} q_{1}+a_{2} q_{1}^{2}}{\sqrt{\alpha}}\right)$

where the value of the integrator gain $\frac{K_{I}}{T}$ is given in Table 1. The actuator parameters in Table 1 are identified experimentally [34]. The natural frequency of the cantilever beam has been found experimentally to be $99.38 \mathrm{kHz}$ and the beam length was determined to be $157 \mu \mathrm{m}$.

\section{Static analysis}

To study the behavior of the actuator, the location of the equilibrium points and their stability conditions are 


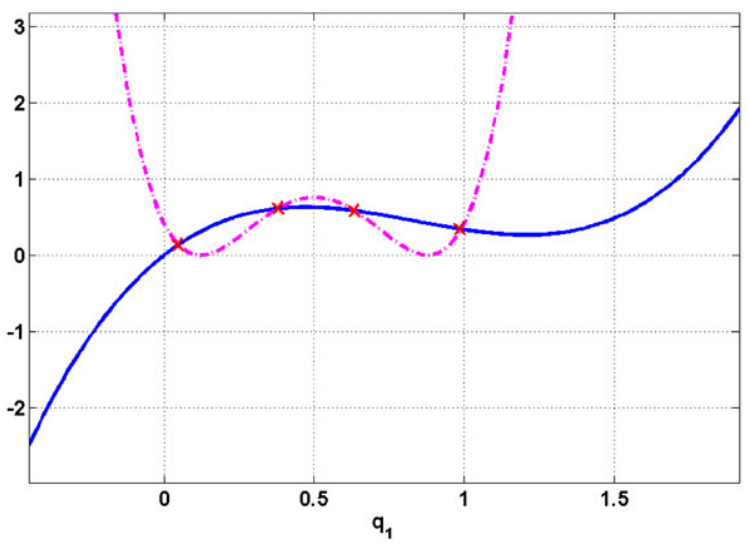

Fig. 3 Graphical solution of (9) showing the balance of the mechanical force and the regulated electrostatic force at $V_{\text {in }}=5 \mathrm{~V}$ for $\frac{a_{0}}{\sqrt{\alpha}}=20.194 \mathrm{~V}, \frac{a_{1}}{\sqrt{\alpha}}=-142.697 \mathrm{~V}$, and $\frac{a_{2}}{\sqrt{\alpha}}=142.697 \mathrm{~V}$. - left side and -.- the middle equation

obtained. The location of the equilibrium points, in the static response, is found by solving the algebraic equation resulting from setting the time derivatives equal to zero in (7):

$$
\begin{aligned}
& \omega_{1}^{2} q\left(1+c_{1} q+c_{2} q^{2}\right) \\
& \quad=c_{3} \alpha\left(V_{\text {in }}-\frac{\left(a_{0}+a_{1} q+a_{2} q^{2}\right)}{\sqrt{\alpha}}\right)^{2}=f .
\end{aligned}
$$

Equation (9) describes the balance of the mechanical force and the regulated electrostatic force. The left hand side of the equation is the spring force multiplied by the denominator of the electrostatic force and the middle equation is the square of the difference of the input voltage and the regulated voltage. The equation can be solved graphically by plotting both sides of the equation as depicted in Fig. 3. As it is seen, the system shows bi-stability as the intersections representing the equilibrium points include two stable and two unstable equilibrium points. The stability conditions are determined by linearizing (7) around the equilibrium points and finding the eigenvalues.

For the system to be bi-stable, the middle quartic equation of (9) in terms of $q$ should have two pairs of roots between 0 and 1 for an assumed $V_{\text {in }}$, as shown by the dash dot line in Fig. 3. These roots then dictate the location of four equilibrium points in the solution of the equation. The farther the roots in each pair are from each other, the closer the first stable and last unstable equilibrium points get to the location of the beam initial position and the fixed electrode, respec-

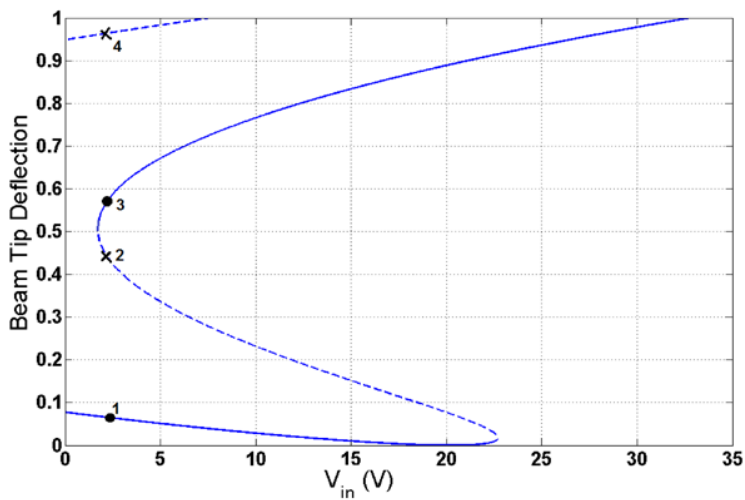

Fig. 4 Non-dimensional deflection of the beam tip versus input DC voltage for $\frac{a_{0}}{\sqrt{\alpha}}=20.194 \mathrm{~V}, \frac{a_{1}}{\sqrt{\alpha}}=-142.697 \mathrm{~V}$, and $\frac{a_{2}}{\sqrt{\alpha}}=142.697 \mathrm{~V},-$ stable, -- unstable

Table 2 Eigenvalues of the equilibrium points at $V_{\text {in }}=2 \mathrm{~V}$

\begin{tabular}{llll}
\hline$\#$ & $q_{1}$ & Eigenvalue 1 & Eigenvalue 2 \\
\hline 1 & 0.066 & $-0.308+5.72 \mathrm{i}$ & $-0.308-5.72 \mathrm{i}$ \\
2 & 0.458 & 2.43 & -3.04 \\
3 & 0.555 & $-0.308+3.02 \mathrm{i}$ & $-0.308-3.02 \mathrm{i}$ \\
4 & 0.962 & 15.89 & -16.51 \\
\hline
\end{tabular}

tively. Assuming the roots, the unknown coefficients of $a_{0}, a_{1}$, and $a_{2}$ are found from solving (9).

The static response of the actuator for the quadratic controller is illustrated in Fig. 4 at different input voltages. The eigenvalues of the equilibrium points at $V_{\text {in }}=2 \mathrm{~V}$ are listed in Table 2. It can be concluded from Fig. 4, and Table 2 that points 1 and 3 are stable equilibrium points and points 2 and 4 are saddles. In other words, the first and the third branches of the static profile are the loci of stable equilibrium points and the second and fourth branches are the loci of the saddles. The upper stable and unstable branches crosses the unity deflection line at $7.4 \mathrm{~V}$, and $32.5 \mathrm{~V}$, respectively. Beyond $32.5 \mathrm{~V}$, the unstable and stable branches diverge from each other in the non-physical domain. The non-dimensional natural frequency of the lower and upper equilibrium points (1 and 3) are 5.72 and 3.02 corresponding to $161.7 \mathrm{kHz}$ and $85.37 \mathrm{kHz}$, respectively.

The closed loop actuator reported [33, 35] consisted of a hyperbolic function $\left(\frac{q}{1-q}\right)$ in contrast to the quadratic function introduced here. Fig. 5 shows the non-dimensional deflection of the closed loop actuator with the hyperbolic function. There exists a region of 


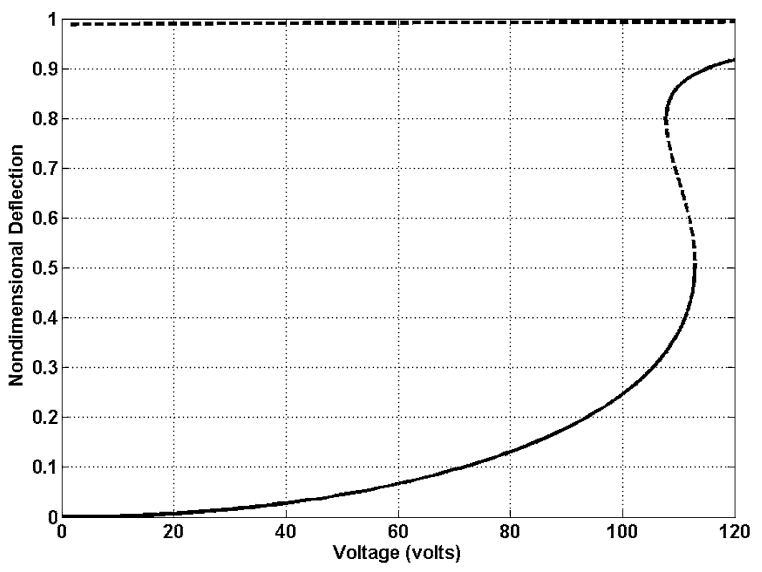

Fig. 5 Non-dimensional deflection of the beam tip versus voltage $V_{\mathrm{DC}}$ for the closed loop actuator with the hyperbolic function reported in [33], - stable, - - unstable

bi-stability and the upper stable branch approaches the unit deflection line asymptotically. The advantages of the quadratic controller (Fig. 4) are wider bi-stability input voltage range and lower bi-stability operating voltage. The bi-stability input voltage region for the quadratic controller extends over $20.95 \mathrm{~V}$ which is four times larger than that of the actuator presented in Fig. 5. The quadratic controller also poses lower bi-stability operating voltages: $1.75 \mathrm{~V}$ to $22.7 \mathrm{~V}$ compared to a minimum operating voltage of $110 \mathrm{~V}$ for the system shown in Fig. 5, thus reducing the requirements on the electronic components in the regulator circuit.

The controller quadratic law is chosen so that the chaotic resonator can be produced both from bistability and from a stable system with an unstable equilibrium placed far from the stable equilibrium point. In other words, the system has four equilibrium points including two stable and two unstable as well as two equilibrium points composed of one stable and one unstable equilibrium point at different ranges of voltage, obtained from solving the static equilibrium (9).

The quadratic controller has also the advantage of tunability in terms of the size of the potential wells of the actuator in the bi-stability region. This property can be seen by in Fig. 4. At low voltages, the upper equilibrium point is close to the lower saddle, while the lower equilibrium point is far from the saddle indicating a large and deep lower well. As the voltage increases, this situation reverses and the upper well

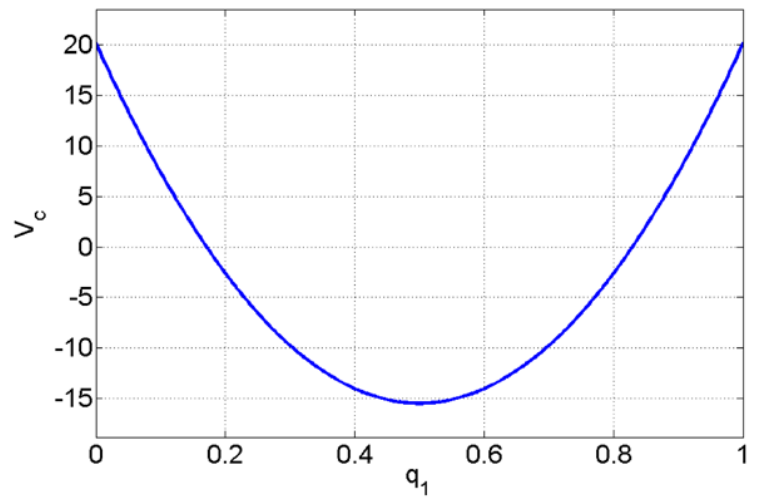

Fig. 6 Equilibrium of controller output voltage $V_{c}$ as a function of the non-dimensional gap when $\frac{a_{0}}{\sqrt{\alpha}}=20.1939 \mathrm{~V}$, $\frac{a_{1}}{\sqrt{\alpha}}=-142.6973 \mathrm{~V}$, and $\frac{a_{2}}{\sqrt{\alpha}}=142.6973 \mathrm{~V}$

becomes larger and deeper as the lower well becomes smaller and shallower.

In terms of implementation, as $q \rightarrow 1$, the proposed quadratic controller circuit is less susceptible to noise than the previous controller [36] by featuring a hyperbolic function $\left(\frac{q}{1-q}\right)$ instead of a quadratic function. The coefficients in the quadratic equation are also chosen to be realizable in an electronic circuit. Further, the controller output voltage $V_{c}$ is limited to a range of $35 \mathrm{~V}(-15 \mathrm{~V}$ to $20 \mathrm{~V})$ for the full actuator motion range, as illustrated in Fig. 6, obtained from solving the equilibrium equation of the controller voltage, (8) with zero time derivatives.

\section{Dynamic analysis}

The steady-state dynamic response of the actuator to a biased harmonic voltage $V_{\text {in }}=V_{\mathrm{DC}}+V_{\mathrm{AC}} \cos \Omega t$ is found by integrating (7) numerically for 5,000 periods of excitation and retaining the last 128 periods. Phase space of dynamic orbits according to (7) consists of three axes: non-dimensional velocity, nondimensional displacement, and controller voltage. The phase portraits found in this section are cross sections of the orbits in the three-dimensional phase space. Bifurcation diagrams are also found revealing the excitation amplitude and frequency ranges where periodic and chaotic vibrations occur.

To take advantage of the dynamic amplification available in the vicinity of primary resonance, we excite at a non-dimensional frequency of 3.2 , corre- 
Fig. 7 (a) Phase portrait of chaotic oscillation, (b) Non-dimensional beam tip displacement,

(c) Controller output

voltage $V_{c}$, for

$\frac{K_{I}}{T}=0.1776 \frac{1}{\mathrm{~s}}$, at

$V_{\mathrm{DC}}=2 \mathrm{~V}, V_{\mathrm{AC}}=1 \mathrm{~V}$, and

$\Omega=90.4 \mathrm{kHz}$ (Crosses

show the locations of

saddles and circles show the

location of nodes.)

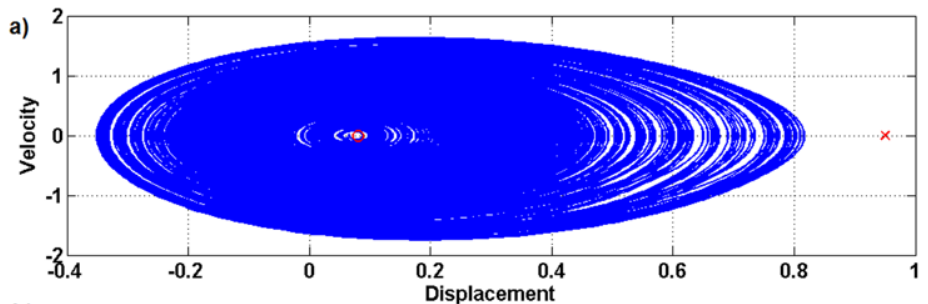

b)

c)
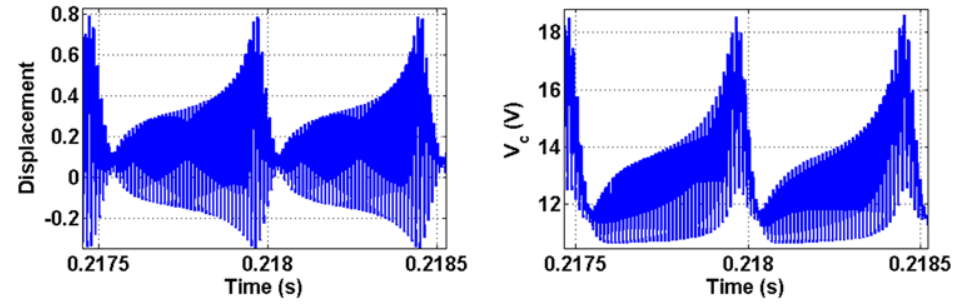

sponding to $90.4 \mathrm{kHz}$, which is slightly above the natural frequency of the upper equilibrium point. It should be noted that due to squared voltage term in (7), the actual excitation frequency is double the nominal excitation frequency. Chaos at this frequency occurs at an $\mathrm{AC}$ amplitude of $V_{\mathrm{AC}}=1 \mathrm{~V}$ (Fig. 7). Figure 7 shows that almost, but not quite, periodic oscillations with short intervals of irregularity develop in the lower well, grow to exceed the potential hump (saddle), and cross to the upper well before being injected back into the lower well to repeat the process. This behavior is typical of an intermittency route to chaos, which is different from the period doubling route to chaos found in the actuator studied in [33]. The chaotic motion developed in this case is mostly in the lower well and between the two wells as the phase portrait and time series of the deflection show. In this case, chaos appears subsequent to a subcritical period-doubling bifurcation. In the former case, chaos appears subsequent to a cascade of supercritical period-doubling bifurcations. The controller voltage is also limited between 10 to $19 \mathrm{~V}$ reduced by more than half of the voltage required by the previous controller [33] to produce same ranges of oscillation.

\subsection{Force sweep}

Observing chaotic attractors mainly in the lower-well led us to seek chaotic attractors that are limited strictly to one well at small DC voltages. Therefore, bifurcation diagrams were obtained from force sweeps at zero DC voltage while keeping the excitation frequency constant.

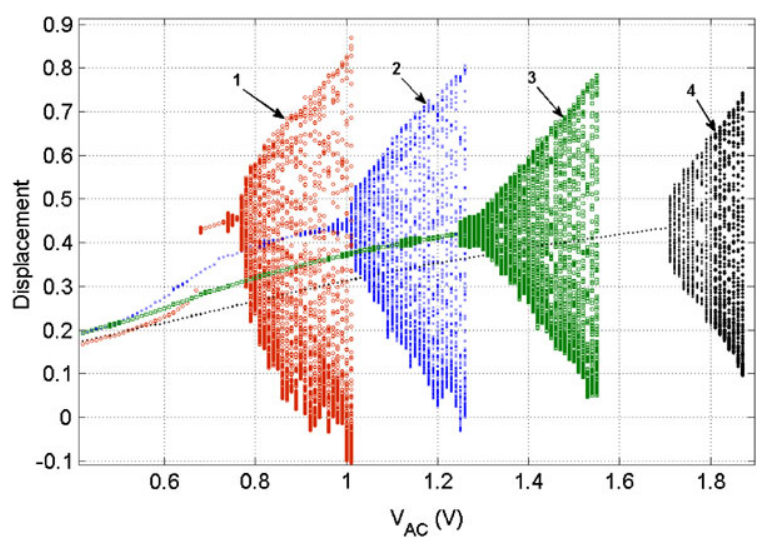

Fig. 8 Bifurcation diagrams constructed by stacking Poincaré sections in force sweeps of $V_{\mathrm{AC}}$ at $V_{\mathrm{DC}}=0$ at four fixed frequencies. 1: $\Omega=90.4 \mathrm{kHz}, 2: \quad \Omega=91.8 \mathrm{kHz}$, 3: $\Omega=93.22 \mathrm{kHz}$, and $4: \Omega=94.64 \mathrm{kHz}$

A single stable equilibrium is available at $V_{\mathrm{DC}}=0$ located at 0.08 of the gap with a natural frequency of $164 \mathrm{kHz}$ (Fig. 4). The bifurcation diagrams in Fig. 8 are obtained for excitation frequencies of $\Omega=90.4 \mathrm{kHz}, \Omega=91.8 \mathrm{kHz}, \Omega=93.22 \mathrm{kHz}$, and $\Omega=94.64 \mathrm{kHz}$. Equations (7) are solved numerically for 5,000 periods of AC excitation and Poincaré section is created by sampling the last 128 periods at the period of excitation starting from the maximum displacement.

The bifurcation diagrams illustrate the progression from periodic motion to intermittent chaos. Chaos disappears beyond a voltage limit where the orbit grows and touches the stable manifold of the upper saddle leading to dynamic pull-in. At the lowest frequency of 
Fig. 9 (a) The phase portrait of chaotic oscillations, (b) non-dimensional beam tip deflection, (c) controller output voltage $V_{c}$, for $\frac{K_{I}}{T}=0.1776 \frac{1}{\mathrm{~s}}$, at $V_{\mathrm{DC}}=0 \mathrm{~V}, V_{\mathrm{AC}}=1 \mathrm{~V}$, and $\Omega=90.4 \mathrm{kHz}$. The cross shows the location of the saddle and the circle shows the location of the stable equilibrium point

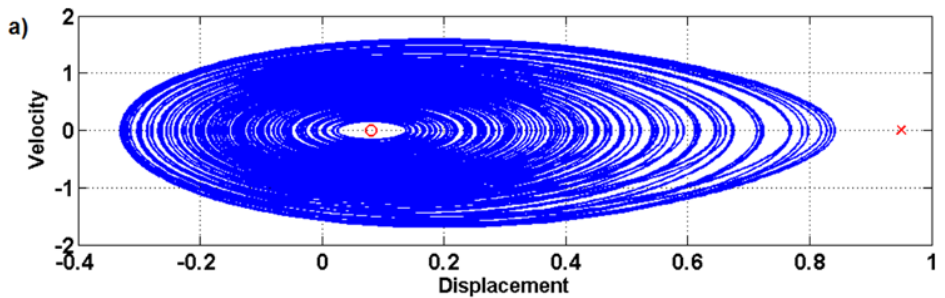

b)

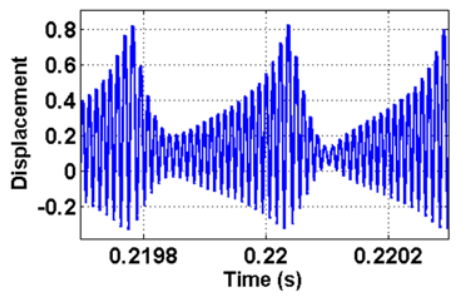

$\Omega=90.4 \mathrm{kHz}$, there is a hysteric jump in the amplitude at $V_{\mathrm{AC}}=0.68 \mathrm{~V}$ before chaos occurs as the $\mathrm{AC}$ voltage increases. The frequency of this is close to the threshold frequency of $89.84 \mathrm{kHz}$ for the appearance of chaos, below this frequency the chaotic oscillations vanish.

The higher the frequency is, the larger the threshold of $\mathrm{AC}$ voltage becomes for the chaotic motions. The reason is that the kinetic energy is higher for higher frequencies making the potential energy and displacement smaller for a constant input energy. That means, orbits at higher frequencies require more voltage to approach the manifold of the saddle and become chaotic. Conversely, the chaotic attractor grows along the displacement axis as the frequency decreases, which is clear comparing the largest attractor at each frequency. For the similar reason, the $\mathrm{AC}$ voltage range for the chaotic motion is the shortest at the largest frequency $\Omega=94.64 \mathrm{kHz}$ shown since more expansion along the velocity axis makes the attractor touch the manifold vertically for a smaller increase in AC voltage. Therefore, to create a chaotic resonator, it is desired to operate as close as possible to the threshold frequency to produce a large displacement signal that makes the detection easier.

The chaotic attractor with the widest footprint along the displacement axis is illustrated in Fig. 9 at the frequency of $90.4 \mathrm{kHz}$ and $V_{\mathrm{DC}}=0 \mathrm{~V}, V_{\mathrm{AC}}=1 \mathrm{~V}$. The displacement signal in part (b) shows vibrations up to $82 \%$ of the gap below and $35 \%$ above the neutral position of the beam. Such large motions are possible by locating the saddle very close to the fixed electrode, and locating the stable equilibrium close to the undeflected beam position (see Fig. 4). It is also noted that even for large oscillations, the controller voltage shown in Fig. 9(c) is limited to the range $8 \mathrm{~V}$ to $17 \mathrm{~V}$. Hence, the quadratic controller made the total displacement signal as large as $117 \%$ of the gap while requiring the controller output voltage as small as $9 \mathrm{~V}$. Using the previous controller, actuation was only feasible up to $90 \%$ of the gap at the cost of twice the controller voltage [35].

Furthermore, such large attractors could indicate the controller robustness as it shows the system can survive the large irregular, but bounded chaotic oscillations covering almost the entire phase space. The quadratic regulator can also control the attractor size and location by appropriate selection of operation point within the excitation parameter space: DC voltage (Fig. 4), AC voltage and frequency (Fig. 8).

Further, the input voltage to the system for large chaotic attractors does not require any DC voltage, compared to $110 \mathrm{~V} \mathrm{DC} \mathrm{(Fig.} \mathrm{5)} \mathrm{for} \mathrm{the} \mathrm{previous} \mathrm{con-}$ troller [33]. Eliminating DC voltage prevents leakage current into the isolation layers of the actuator and avoids drift of the measured parameter values over time.

\subsection{Frequency sweep}

The frequency response curves shown in Figs. 10 and 11 are obtained by sweeping the frequency of excitation at two fixed $\mathrm{AC}$ voltages of $V_{\mathrm{AC}}=1.23 \mathrm{~V}$ and $V_{\mathrm{AC}}=1 \mathrm{~V}$, respectively. The results are obtained by solving (7) numerically for 5,000 periods of AC excitation. Poincaré sections are obtained by sampling 


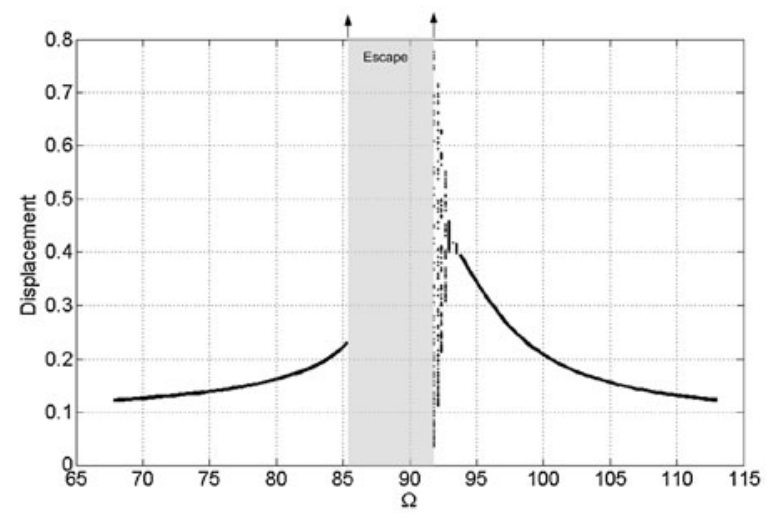

Fig. 10 Bifurcation diagram constructed by stacking Poincaré sections in a frequency sweep at $V_{\mathrm{AC}}=1.23 \mathrm{~V}$ and $V_{\mathrm{DC}}=0$

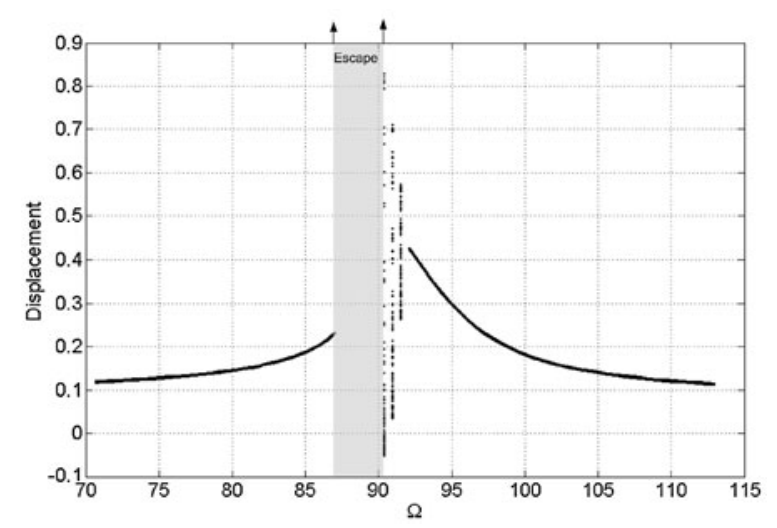

Fig. 11 Bifurcation diagram constructed by stacking Poincaré sections in a frequency sweep at $V_{\mathrm{AC}}=1 \mathrm{~V}$ and $V_{\mathrm{DC}}=0$

the last 128 periods at the period of excitation starting from the maximum displacement. Decreasing the excitation frequency in the first case from $113 \mathrm{kHz}$, the period-one orbit grows until chaos appears through an intermittency route at $93.51 \mathrm{kHz}$ and continues down to $91.8 \mathrm{kHz}$. Below that, the chaotic attractor becomes very large, touches the stable manifold of the saddle, and goes to dynamic pull-in, in a process known as the escape from a potential well [37]. The escape region, where there is no solution, persists down to the frequency of $85.3 \mathrm{kHz}$, below which the system response continues to be periodic with small period-one orbits. Operation in this region should be avoided since it will lead to the moving electrode losing stability and coming into contact with the fixed electrode.

Both frequency sweeps reveal softening-type response with a small chaotic region. Comparing the two bifurcation diagrams, the escape region shrinks,

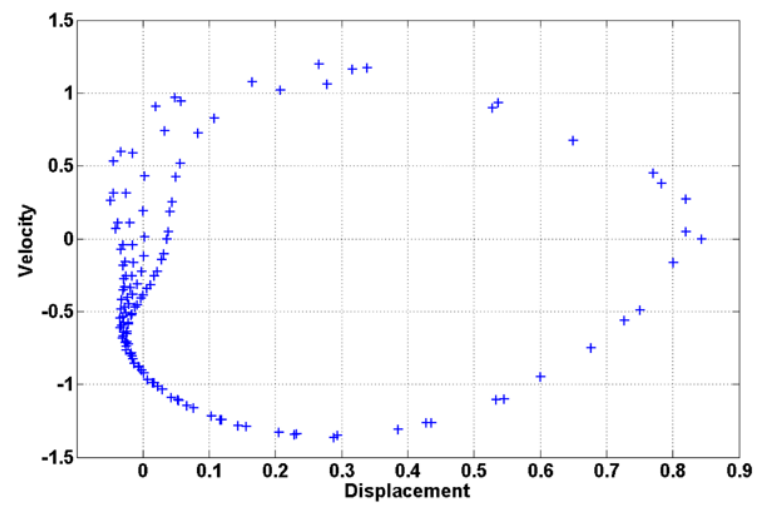

Fig. 12 Poincaré section for the attractor in Fig. 9 starting from initial conditions of $\left[q_{0}=0, \dot{q}_{0}=0, V_{c 0}=0\right]$

the threshold frequency for chaos drops, while the largest attractor grows for lower AC excitation. Lowering down the AC excitation voltage eventually eliminates the escape region and the chaotic region to be replaced by multi-valued periodic responses and hysteric jumps observed in the force sweep start to appear. The largest chaotic attractors shown in Figs. 10 and 11 correspond to the largest attractors in Fig. 8 for frequencies of $91.8 \mathrm{kHz}$ and $90.4 \mathrm{kHz}$, respectively.

\subsection{Information entropy}

Information entropy is used as a metric to verify the chaotic attractors observed in this study. The size of a box that surrounds the chaotic attractor in the three dimensional phase space (non-dimensional velocity, non-dimensional displacement, and controller voltage) is obtained and is divided to small cubes of size $\epsilon=$ $0.0002 \mathrm{~V}$. Then a Poincare section of the attractor is found by sampling at the period of excitation starting from the maximum displacement. The number of points in each cube is counted $\left(N_{i}\right)$ and divided by the total number of points on the Poincaré section $\left(N_{0}\right)$ to get $P_{i}=\frac{N_{i}}{N_{0}}$. The information entropy is then calculated as [38]:

$I(\epsilon)=-\sum^{N} P_{i} \log P_{i}$

where $N$ is the total number of cubes. The system is fully predictable when all points are located in one cell and, therefore, $I=0$. Any value between 0 and 1 is a measure of the unpredictability of a chaotic attractor.

The Poincaré section of the attractor shown in Fig. 9 is illustrated in Fig. 12. It clearly shows onset of 


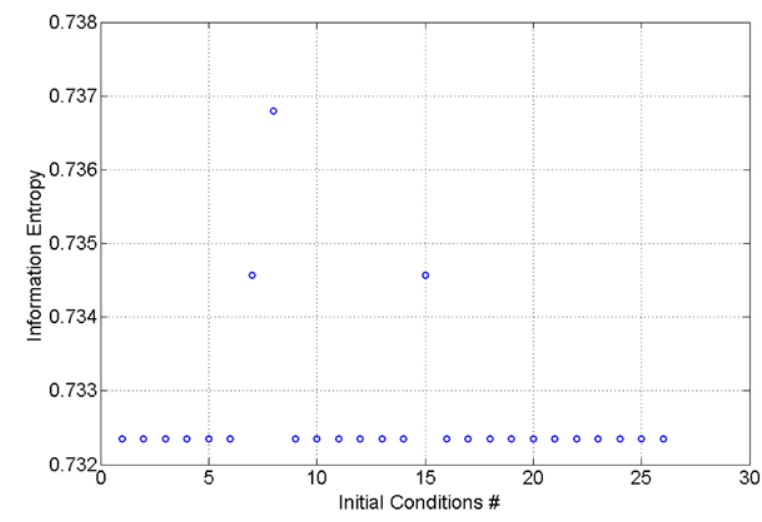

Fig. 13 Information entropy for the chaotic attractor at $\frac{K_{I}}{T}=0.1776 \frac{1}{s}, V_{\mathrm{DC}}=0 \mathrm{~V}, V_{\mathrm{AC}}=1 \mathrm{~V}$, and $\Omega=90.4 \mathrm{kHz}$. The initial conditions are $q_{0}$ at equal increments between $[0.28-0.66], \dot{q}_{0}=0.1$, and $V_{c 0}=0 \mathrm{~V}$

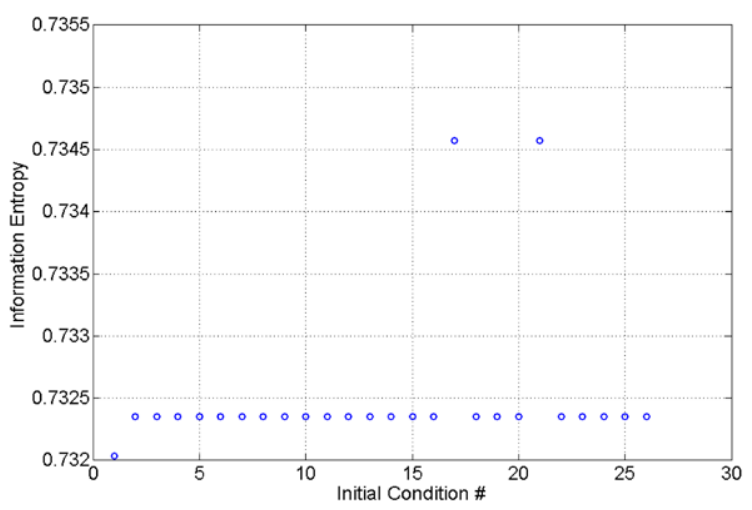

Fig. 14 Information entropy for the chaotic attractor at $\frac{K_{I}}{T}=0.1776 \frac{1}{\mathrm{~s}}, V_{\mathrm{DC}}=0 \mathrm{~V}, V_{\mathrm{AC}}=1.8 \mathrm{~V}$, and $\Omega=94.64 \mathrm{kHz}$. The initial conditions are $q_{0}$ at equal increments between $[0.28-0.66], \dot{q_{0}}=0.1$, and $V_{c 0}=0 \mathrm{~V}$

chaos as the return-map fill an area rather than discrete points or an orbit. The information entropy is obtained for the attractor starting from different initial conditions in the phase space as presented in Fig. 13. Information entropies are found to vary between 0.732 and 0.737 , which proves that the oscillations are chaotic. The figure also reveals that there is a common information entropy of 0.7324 for 23 out of 26 initial conditions with a small variation of less than 0.005 for the other three initial conditions.

Information entropy is also found for an attractor located at $V_{\mathrm{AC}}=1.8 \mathrm{~V}$ and $\Omega=94.64 \mathrm{kHz}$ in Fig. 8 using the same initial conditions of Fig. 13. The calculated information entropies are illustrated in Fig. 14, which shows a common value of 0.732 for 23 out of
26 initial conditions and small variation for the other three initial conditions. The information entropy was also obtained for other attractors and it was observed that there was a dominant value of 0.732 for all attractors that does not depend on the excitation frequency or amplitude indicating a unique chaotic characteristic.

\section{Summary and future work}

A voltage regulator with a quadratic control function is developed for an electrostatic actuator to make a large oscillator. Bifurcation diagrams obtained by sweeping the magnitude and frequency of excitation reveal periodic and large bounded chaotic oscillation regions in the parameter space. The quadratic controller demonstrates valuable advantages over the previously studied controller. Not only the quadratic regulator improves the system handling of noise, but also it requires no input DC voltage that avoids leakage current in the isolation layers of MEMS. The regulator drives the beam to vibrate in the positive and negative directions for a total range of $117 \%$ of the gap. It requires only half of the previous regulator output voltage, thus decreasing the load on the circuit components. The large oscillations of the quadratic resonator are important as they are bounded in the phase space, thus making detection easier which suitable for use in sensing applications. Furthermore, the resonator is potentially robust as it demonstrates a stable response over a large area of the phase space.

The regulator has two potential functions: one-well once the DC and RMS of AC voltage is below a threshold, and two-well once this value is above the threshold. Both potential functions can be used for linear or non-linear oscillators depending on the purpose. The one-well potential field allows creation of an oscillator with a large chaotic attractor. The two-well potential field allows creation of both a relatively small onewell attractor protected by the upper well and a large two-well attractor. The size of the chaotic attractors are also controllable setting the frequency of excitation.

Finally a metric of chaos, namely information entropy, is used to prove that the observed long period orbits are indeed chaotic and to measure their irregularity. It was found the chaotic attractors have a dominant information entropy of 0.732 that does not depend on the excitation frequency and magnitude and is 
a characteristic of the chaotic process in the quadratic resonator.

In order to realize a prototype for the controller in the future work, a similar approach used by the authors [36] to make the hyperbolic controller can be applied. The controller was implemented using an analog circuit. In that study, to measure the deflection used by the circuit, a laser Doppler vibro-meter was used to measure the velocity at the tip of the moving electrode which was then integrated to obtain the tip displacement. For a controller prototype, the same approach can be used here to measure the beam tip displacement and to use that signal in an analog circuit controller with a quadratic function. For practical implementation of a real system, an approach that can measure the phase difference between input voltage and output voltage is probably more suitable than capacitive sensing, since large parasitic capacitance will make impede measurements highly inaccurate.

Acknowledgements The authors would like to acknowledge the Natural Sciences and Engineering Research Council of Canada (NSERC) for a Ph.D. postgraduate scholarship.

\section{References}

1. Epureanu, B.I., Tang, L.S., Paidoussis, M.P.: Exploiting chaotic dynamics for detecting parametric variations in aeroelastic systems. AIAA J. 42(4), 728-735 (2004)

2. Epureanu, B.I., Yin, S.-H.: Identification of damage in an aeroelastic system based on attractor deformations. Comput. Struct. 82(31-32), 2743-2751 (2004)

3. Epureanu, B.I., Yin, S.H., Derriso, M.M.: High-sensitivity damage detection based on enhanced nonlinear dynamics. Smart Mater. Struct. 14(2), 321-327 (2005)

4. Ghafari, S.: A fault diagnosis system for rotary machinery supported by rolling element bearings. $\mathrm{PhD}$ thesis, University of Waterloo (2007)

5. Yin, S.-H., Epureanu, B.I.: Experimental enhanced nonlinear dynamics and identification of attractor morphing modes for damage detection. J. Sound Acoust. 129(6), 763770 (2007)

6. Cuomo, K.M., Oppenheim, A.V., Strogatz, S.H.: Synchronization of Lorenz-based chaotic circuits with applications to communications. In: IEEE Transactions on Circuits and Systems II: Analog and Digital Signal Processing, vol. 40, pp. 626-633 (1993)

7. Park, K., Chen, Q., Lai, Y.C.: Energy enhancement and chaos control in microelectromechanical systems. Phys. Rev. 77, 026210-1-6 (2008)

8. Buks, E., Yurke, B.: Mass detection with a nonlinear nanomechanical resonator. Phys. Rev. E 74(4), 46619-4619 (2006)
9. Conley, W.G., Raman, A., Krousgrill, C.M., Mohammadi, S.: Nonlinear and nonplanar dynamics of suspended nanotube and nanowire resonators. Nano Lett. 8(6), 1590-1595 (2008)

10. Chen, Q., Huang, L., Lai, Y.C., Grebogi, C., Dietz, D.: Extensively chaotic motion in electrostatically driven nanowires and applications. Nano Lett. 10(2), 406-413 (2010)

11. Basso, M., Giarré, L., Dahleh, M., Mezic, I.: Numerical analysis of complex dynamics in atomic force microscopes. In: Proceedings of IEEE International Conference on Control Applications, Trieste, Italy, pp. 1026-1030 (1998)

12. Ashhab, M., Salapaka, M.V., Dahleh, M., Mezic, I.: Melnikov-based dynamical analysis of microcantilevers in scanning probe microscopy. Nonlinear Dyn. 20(3), 197220 (1999)

13. Ashhab, M., Salapaka, M.V., Dahleh, M., Mezic, I.: Dynamical analysis and control of microcantilevers. Automatica 35(10), 1663-1670 (1999)

14. Lee, S.I., Howell, S.W., Raman, A., Reifenberger, R.: Nonlinear dynamics of microcantilevers in tapping mode atomic force microscopy: A comparison between theory and experiment. Phys. Rev. B, Condens. Matter Mater. Phys. 66(11), 115409/1-10 (2002)

15. Raman, A., Hu, S.: Chaos in dynamic atomic force microscopy. In: 2006 International Symposium on Nonlinear Theory and Its Applications, Bologna, Italy, pp. 911-914 (2006)

16. Jamitzky, F., Stark, M., Bunk, W., Heckl, W.M., Stark, R.W.: Chaos in dynamic atomic force microscopy. Nanotechnology 17(7), S213-S220 (2006)

17. Bienstman, J., Vandewalle, J., Puers, R.: Autonomous impact resonator: A new operating principle for a silicon resonant strain gauge. Sens. Actuators A, Phys. 66(1-3), 40-49 (1998)

18. Wang, Y.C., Adams, S.G., Thorp, J.S., MacDonald, N.C., Hartwell, P., Bertsch, F.: Chaos in MEMS, parameter estimation and its potential application. IEEE Trans. Circuits Syst. I, Fundam. Theory Appl. 45(10), 1013-1020 (1998)

19. De, S.K., Aluru, N.R.: Complex nonlinear oscillations in electrostatically actuated microstructures. J. Microelectromech. Syst. 15(2), 355-369 (2006)

20. DeMartini, B.E., Butterfield, H.E., Moehlis, J., Turner, K.L.: Chaos for a microelectromechanical oscillator governed by the nonlinear Mathieu equation. J. Microelectromech. Syst. 16(6), 1314-1323 (2007)

21. Zhang, Y., Wang, Y.S., Li, Z.H., Huang, Y.B., Li, D.C.: Snap-through and pull-in instabilities of an arch-shaped beam under an electrostatic loading. J. Microelectromech. Syst. 16(3), 684-693 (2007)

22. Krylov, S.: Lyapunov exponents as a criterion for the dynamic pull-in instability of electrostatically actuated microstructures. Int. J. Non-Linear Mech. 42(4), 626-642 (2007)

23. Krylov, S., Ilic, B.R., Schreiber, D., Seretensky, S., Craighead, H.: The pull-in behavior of electrostatically actuated bistable microstructures. J. Micromech. Microeng. 18(5), 1-20 (2008)

24. Das, K., Batra, R.C.: Pull-in and snap-through instabilities in transient deformations of microelectromechanical systems. J. Micromech. Microeng. 19(3), 1-19 (2009) 
25. Rhoads, J.F., Shaw, S.W., Moehlis, J., Demartini, B.E., Turner, K.L., Zhang, W.: Nonlinear response of parametrically-excited MEMS. In: DETC2005: ASME International Design Engineering Technical Conferences and Computers and Information in Engineering Conference, vol. 1A, pp. 453-461 (2005)

26. Zhang, W.-M., Meng, G.: Nonlinear dynamic analysis of electrostatically actuated resonant MEMS sensors under parametric excitation. IEEE Sens. J. 7(3), 370-380 (2007)

27. Liu, S., Davidson, A., Lin, Q.: Simulation studies on nonlinear dynamics and chaos in a MEMS cantilever control system. J. Micromech. Microeng. 14(7), 1064-1073 (2004)

28. Younis, M.I., Ouakad, H., Alsaleem, F.M., Miles, R., Cui, W.: Nonlinear dynamics of MEMS arches under harmonic electrostatic actuation. J. Microelectromech. Syst. 19(3), 647-656 (2010)

29. Ouakad, H., Younis, M.I.: The dynamic behavior of MEMS arch resonators actuated electrically. Int. J. Non-Linear Mech. 45(7), 704-713 (2010)

30. Lu, M.S.-C., Fedder, G.K.: Position control of parallel-plate microactuators for probe-based data storage. J. Microelectromech. Syst. 13(5), 759-769 (2004)

31. Alsaleem, F.M., I Younis, M.: Integrity analysis of electrostatically actuated resonators with delayed feedback con- troller. J. Dyn. Syst. Meas. Control 133(3), 031011 (2011) (8 pages)

32. Alsaleem, F.M., Younis, M.I.: Stabilization of electrostatic MEMS resonators using delayed feedback controller. Smart Mater. Struct. 19, 035016 (2010)

33. Towfighian, S., Heppler, G.R., Abdel-Rahman, E.M.: Analysis of a chaotic electrostatic micro-oscillator. J. Comput. Nonlinear Dyn. 6(1), 011001-10 (2011)

34. Towfighian, S., Heppler, G.R., Abdel-Rahman, E.M.: A low voltage controller for a chaotic micro resonator. In: Proceedings of the ASME 2010 International Design Engineering Technical Conferences. pp. DETC2010-28990, Montreal, QC, Canada (2010)

35. Towfighian, S.: A Large-stroke electrostatic micro-actuator. $\mathrm{PhD}$ thesis, University of Waterloo (2010)

36. Towfighian, S., Seleim, A., Abdel-Rahman, E.M., Heppler, G.R.: A large stroke electrostatic micro-actuator. J. Micromech. Microeng. 21, 075023 (2011) (12 pages)

37. Younis, M.I., Alsaleem, F.: Exploration of new concepts for mass detection in electrostatically-actuated structures based on nonlinear phenomena. J. Comput. Nonlinear Dyn. 4(2), 021010-1-15 (2009)

38. Moon, F.C.: Chaotic Vibrations: An Introduction for Applied Scientists and Engineers. Wiley-Interscience, New York (1987) 